\title{
In Vivo Therapeutic Effects of Four Synthesized Antileishmanial Nanodrugs in the Treatment of Leishmaniasis
}

\author{
Tahereh Zadeh Mehrizi ${ }^{1}$, Nariman Mosaffa ${ }^{2}$, Mostafa Haji Molla Hoseini ${ }^{2}$, Mehdi Shafiee \\ Ardestani ${ }^{3{ }^{3 * *}}$, Ali Khamesipour ${ }^{4}$, Hasan Ebrahimi Shahmabadi ${ }^{5}$, Mostafa Pirali Hamedani ${ }^{6}$, Yasaman \\ Mardani Dashti ${ }^{7}$ and Amitis Ramezani ${ }^{1,{ }^{*}}$ \\ ${ }^{1}$ Department of Clinical Research, Pasteur Institute of Iran, Tehran, Iran \\ ${ }^{2}$ Department of Medical Immunology, School of Medicine, Shahid Beheshti University of Medical Sciences, Tehran, Iran \\ ${ }^{3}$ Department of Radiopharmacy, Faculty of Pharmacy, Tehran University of Medical Sciences, Tehran, Iran \\ ${ }^{4}$ Center for Research and Training in Skin Diseases and Leprosy, Tehran University of Medical Sciences, Tehran, Iran \\ ${ }^{5}$ Department of Microbiology, Faculty of Medicine, Rafsanjan University of Medical Sciences, Rafsanjan, Iran \\ ${ }^{6}$ Department of Pharmacognosy, Faculty of Pharmacy, Tehran University of Medical Sciences, Tehran, Iran \\ Islamic Azad University, Medical Branch, Tehran, Iran \\ "Corresponding author: Department of Clinical Research, Pasteur Institute of Iran, Tehran, Iran. Tel: +98-2164112812, E-mail: ametesramezani@gmail.com \\ Corresponding author: Department of Radiopharmacy, Faculty of Pharmacy, Tehran University of Medical Sciences, Tehran, Iran. Tel: +98-9128267629, E-mail: \\ shafieeardestani@gmail.com
}

Received 2018 June 09; Revised 2018 September 01; Accepted 2018 September 09.

\begin{abstract}
Background: Nowadays, nanocarriers are used for leishmaniasis treatment due to development of drug resistance and several side effects with conventional therapeutics.

Objectives: In this study we aimed to evaluate in vivo effects of four synthesized nanodrugs including amphotericin B-nanochitosan (AK), betulinic acid-nanochitosan (BK), amphotericin B-dendrimer(AD), and betulinic acid-dendrimer (BD) in the treatment of Leishmania major infection (L. major) in mice model by using pathological analyses to choose the most effective nanodrug in leishmaniasis.

Methods: The four nanodrugs efficacy in the improvement of L. major lesion in a mice model was evaluated by using pathological analyses including measurement of organs size and parasite number. Additionally, the nanodrugs toxicity was evaluated by measurement of various blood factors.

Results: The histopathological results of the present study showed that BK, at the dose of $20 \mathrm{mg} / \mathrm{kg}$, and AK, at the dose of $10 \mathrm{mg} / \mathrm{kg}$, were more effective in decreasing the parasite number in the kidney, liver, and spleen. Moreover, BK20 mg/kg and AK10 mg/kg decreased the organs size significantly while AD50 mg/kg and BD40 mg/kg were less effective. However, none of the four nanodrugs had increased the blood factors and they were not toxic.

Conclusions: Overall, the pathologic findings of various mice organs treated with different formulations showed that AK10 mg/kg and BK20 mg/kg were more effective in recovery of L. major's pathological effects in comparison to AD50 mg/kg and BD40 mg/kg. Therefore, it seems that $A K$ and $B K$, in this mentioned dosage, could be considered as a proper candidate for treatment of leishmaniasis.
\end{abstract}

Keywords: Histopathology, L. major, Nanochitosan, Nanodendrimer, Amphotericin B, Betulinic Acid

\section{Background}

Leishmania major (L. major) is the most common species that causes leishmaniasis in Iran $(1,2)$. However, leishmaniasis treatment had encountered several challenges such as limited number of therapeutic agents, side effects, and development of drug resistance (3). Pentavalent antimonials, amphotericin $\mathrm{B}(A)$, and pentamidine are active against $L$. major, however, with several adverse reactions $(2,3)$. Moreover, in recent years, antileishmanial ef- fect of betulinic acid (B) has been shown (4).

Recently, nanotechnology was considered as an appropriate alternative due to the fact that we can deliver current medicines by nanosized carriers and improve bioavailability and reduce toxicity (5). In this regard, researchers have used two nanocarriers of nanochitosan (K) and anionic linear globular dendrimer (ALGD) for treatment of leishmaniasis to decrease the side effects of conventional medicines (6-13). Nanochitosan is a nanocarrier with ability to decrease the drug toxicity and increase the

Copyright (c) 2018, Archives of Clinical Infectious Diseases. This is an open-access article distributed under the terms of the Creative Commons Attribution-NonCommercial 4.0 International License (http://creativecommons.org/licenses/by-nc/4.0/) which permits copy and redistribute the material just in noncommercial usages, provided the original work is properly cited. 
drug solubility. It has additionally immunomodulatory and wound healing effects and was synthesized with various methods including ionic gelation, phase separation, etc. $(10,14)$. Some studies applied nanoformulation of amphotericin B-nanochitosan (AK) synthesized by ionic gelation on leishmaniasis treatment $(7-10,12,13,15,16)$. Furthermore, dendrimer is a nanocarrier with ability to increase the drug solubility. Studies demonstrated that A was loaded into positive charge dendrimers that could increase drug solubility and reduce drug adverse effects (6, $11,17)$. However, both of these nanocarriers had low effects on leishmaniasis lesions and it seems that all researchers used low doses of AK and amphotericin B-dendrimer (AD) for treatment $(7-10,12,13,15,16)$.

In the present study, for the first time, we used higher doses of amphotericin B in both nanocarriers for improving efficacy of our medicines in the mice model. Then, we evaluated therapeutic effects of four nanodrugs AK, betulinic acid-nanochitosan (BK), AD, and betulinic aciddendrimer (BD) on L. major infection by using pathological analyses (measurement of organs size and parasite number) and serum enzymes measurement to choose the most effective nanodrug in leishmaniasis treatment.

\section{Methods}

2.1. In Vivo Toxicity of Nanodrugs by Measuring the Blood Factors

For this purpose, female Balb/c mice were purchased from the Pasteur Institute of Iran. The animals were kept in an animal house under a $12 \mathrm{~h} / 12 \mathrm{~h}$ light/dark cycle, at constant temperature of $22^{\circ} \mathrm{C}$ and $50 \%$ - 60\% humidity condition. They had free access to food and water. All animal experiments were approved by the animal ethics committee of Pasteur Institute of Iran. Based on the protocol, one week later, animals were randomly divided into several groups and after challenging with different doses of each nanodrug and selecting an appropriate one as AK10, BK20, AD50, BD40, GUL200 mg/kg, the toxicity effects were evaluated by measurement of blood tests and the findings were compared to those results obtained from control groups including A50, B40 mg/kg groups, vehicle control, and healthy groups of mice (without receiving any nanodrug). All nanodrugs were administered intraperitoneally. $\mathrm{B}(\mathrm{B}, \mathrm{BD}, \mathrm{BK})$ and $\mathrm{A}(\mathrm{A}, \mathrm{AD}, \mathrm{AK})$ containing formulations were administered every other day for 41 and 21 days retrospectively. After that, animals were anesthetized and the blood samples were obtained. Then, the concentrations of blood factors including blood urea nitrogen (BUN), creatinine (CR), aspartate aminotransferase (AST), alanine aminotransferase (ALT), and alkaline phosphatase (ALP) were measured spectrophotometrically.
2.2. The Organs Size Measurement in Mice Treated with the Nanodrugs

At first, the $L$. major infected $\mathrm{Balb} / \mathrm{c}$ mice were developed (18) and after developing lesion, the treatment process was initiated. The mice were randomly divided into 10 groups including A(A50, AK10, AD50), B (B40, BK20, BD40), K35.5, D12.5, GUL200 mg/kg and negative control. A (A, AK, $\mathrm{AD})$ and $\mathrm{B}(\mathrm{B}, \mathrm{BK}, \mathrm{BD})$ containing formulations were administered intraperitoneally every other day for 21 days and 41 days retrospectively. Additionally, the animals received K, $\mathrm{D}$, and GUL as B containing formulations administration protocol. All formulations were administered intraperitoneally and in the volume of $50 \mu \mathrm{L}$. After that, the animals were sacrificed and liver, kidney, footpad, and spleen were harvested and immersed into $10 \%$ formalin as fixator. Next, the organs size was measured by using pathological ruler.

\subsection{The Parasite Number Measurement in Liver, Kidney, Spleen and Footpad}

For pathologic examination, all mentioned organs were then embedded in paraffin and tissue sections $(5 \mu \mathrm{m})$ were stained with hematoxylin and eosin (HEE) as the general staining and giemsa as the specific staining method. The number of amastigotes were calculated in four sections in each organ by using light microscopy with $60 \mathrm{X}$ magnification (19).

\section{Results}

3.1. The Results of In Vivo Toxicity of the Nanodrugs by Measuring the Blood Factors

No toxicity was found in negative (healthy) and positive control (GUL200) and also solvent receiver (vehicle) group of mice. Furthermore, the nanodrugs (AK, BK, AD, $B D)$ receiving groups did not show any toxicity, whereas at the same dose, the $A$ and $B$ drugs groups showed significant increase of BUN, CR, ALT, AST, and ALP blood tests (Figure 1). The results are expressed as mean \pm SD from three independent experiments.

\subsection{The Results of Organs Size Measurement in Mice Treated with Nanodrugs}

The results showed that L. major infected Balb/c mice received only PBS (negative control) and dendrimer group had the largest organs (liver, spleen and foot pad except kidney) size compared to other groups. However, A, B, and glucantime receiving groups showed slightly smaller size of infected organs, however, the size of these organs in $\mathrm{AD}$ and $\mathrm{BD}$ groups were decreased remarkably. Nonetheless, interesting findings were observed in AK and BK receiver mice, which were recovered completely and organ size were similar to those of the healthy group (Figure 2). 

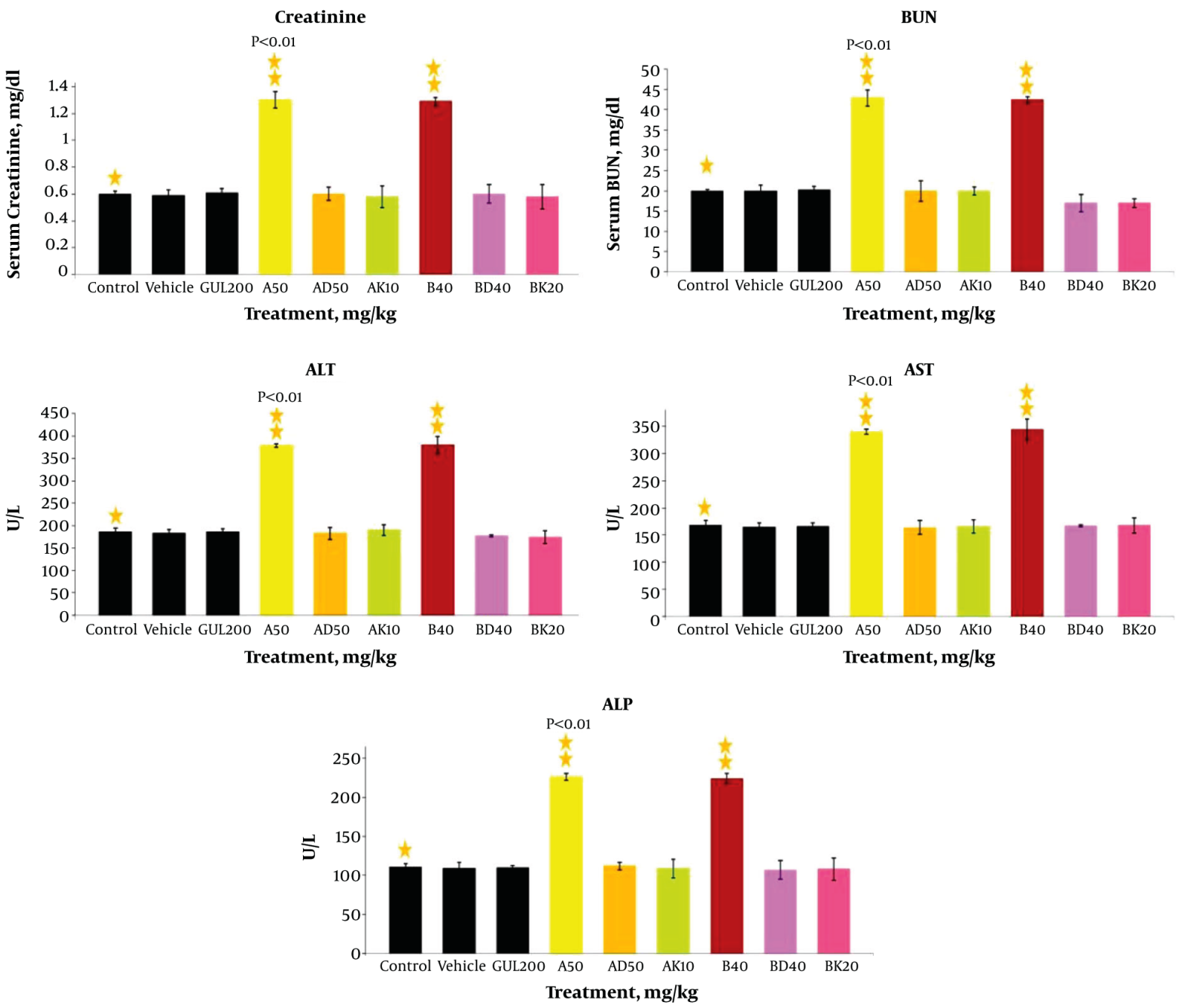

Figure 1. The serum concentrations of BUN, CR, ALT, AST, and ALP in various groups of mice were treated with different formulations. The results are expressed as mean \pm SD from three independent experiments.

Regarding kidney, no enlargement was observed in study groups' samples, except color change, which was reported only in PBS and D groups.

Totally, the results of organ size measurement showed that $\mathrm{AK}$ and $\mathrm{BK}$, in dosage of $10 \mathrm{mg} / \mathrm{kg}$ and $20 \mathrm{mg} / \mathrm{kg}$ retrospectively, were effective in improvement of pathologic effects of leishmaniasis.

3.3. Histopathological Results of Parasite Number in Mice Received Different Formulations

The results of parasite number measurement in different organs and groups of mice were as follow:

- Liver, Spleen, and Footpad: The parasite numbers in different tissues of negative control group were liver: $6 \pm$
2, spleen: $4 \pm 1$, footpad: $18 \pm 6$ amastigotes.

- GUL, A, and B groups showed the same parasite number as liver: $4 \pm 1$, spleen: $3 \pm 1$, and footpad: $6 \pm 3$.

- In K and D groups showed liver: $5 \pm 2$, spleen: $4 \pm 1$, and footpad: $7 \pm 3$ amastigotes while in the groups of $\mathrm{AD}$ and $\mathrm{BD}$, we only detected $1 \pm 1$ parasite in each organ.

- AK and BK groups were considered as a complete recovery group due to the fact that we did not find any parasite in all organs (Figure 3).

- Kidney: The parasite number of $4 \pm 1$ was seen in L. major infected control group, while there was no parasite in the kidney of other treated groups (Figure 3).

Therefore, AK and BK were the most effective formulations for inhibition of amastigotes in various tissues in- 

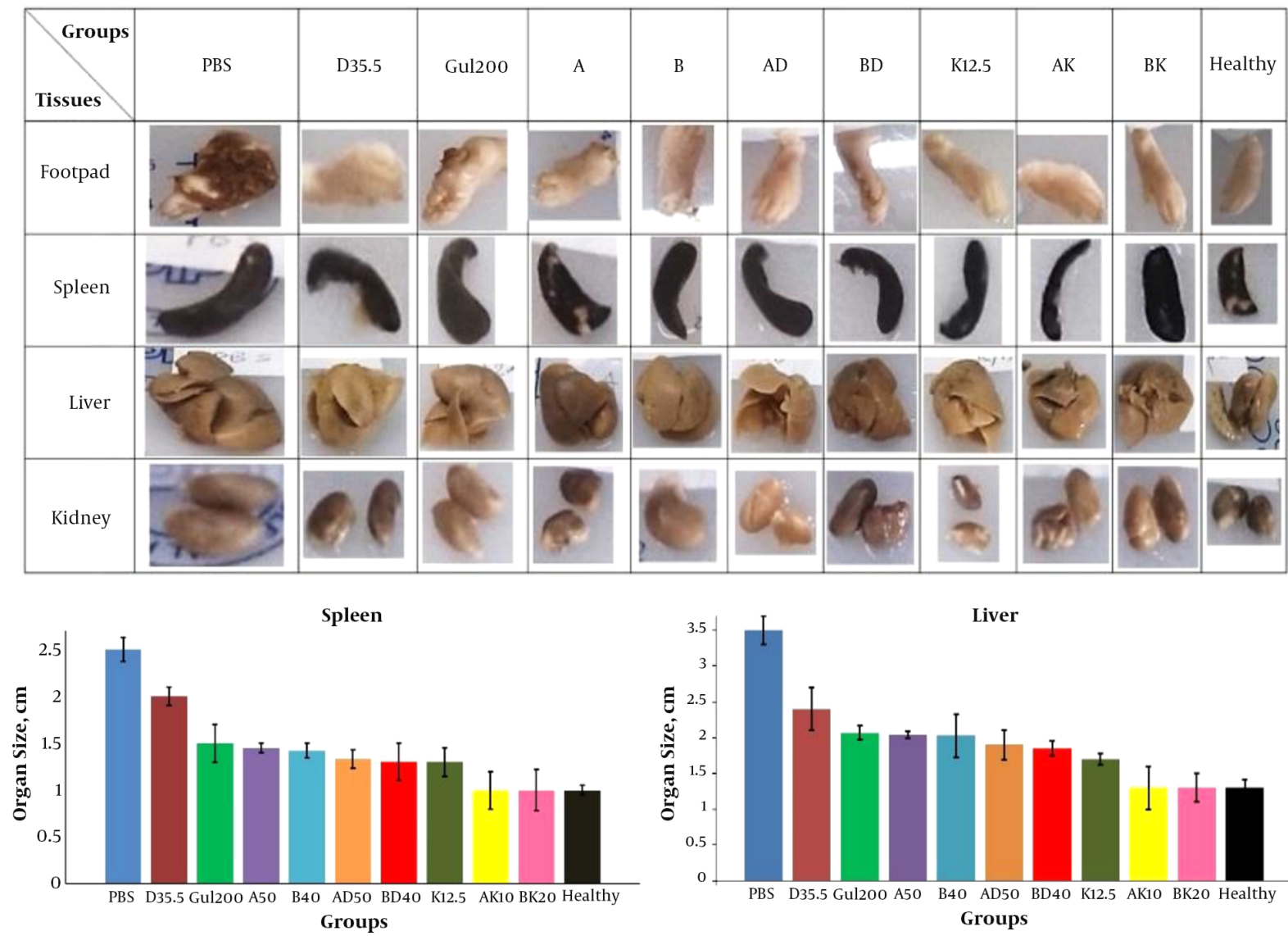

Figure 2. The size of footpad, spleen, liver, and kidney in mice received different formulations (upper part of the figure). In the lower part of the figure, the graphs of spleen and liver size $(\mathrm{cm})$ were showed. The results are expressed as mean $\pm S D$ from three independent experiments.

fected with leishmania.

\section{Discussion}

In this study we evaluated the therapeutic effects of four nanodrugs on leishmaniasis. Pathologic findings showed that AK10 $\mathrm{mg} / \mathrm{kg}$ and BK20 mg/kg were more effective in recovery of $L$. major's pathological effects in comparison to $\mathrm{AD} 50 \mathrm{mg} / \mathrm{kg}$ and $\mathrm{BD} 40 \mathrm{mg} / \mathrm{kg}$ in the mice model. Therefore, it seems that $A K$ and $B K$, in this mentioned dosage, could be considered as a proper candidate for treatment of leishmaniasis.

Current therapies used for leishmaniasis encountered some difficulties due to their high toxicity and low water solubility $(8,10,20-23)$. Nowadays nanocarriers were successful in solving these problems (5).

Some researchers used $\mathrm{K}$ and D nanocarriers for increasing the drug potency, solubility rate, and decreasing drugs side effects (6-13, 15-17, 24). In a study, $A$ was loaded into Polyamidoamine (PAMAM) as a positive charge D and the results showed that PAMAM (D) increased the solubility rate of the drug and decreased its toxicity effects. Moreover, they showed that dendrimers with positive charge (PAMAM and PPI) were not effective in inhibiting parasite and may be due to using low therapeutic dose of A in vivo environment $(6,11,17)$.

Chitosan is an antileishmanial nanocarrier by itself and is used as a carrier for these medicine. The studies of Tripathi et al., Ribeiro et al., and Singh et al., reported that the loading of $A$ into chitosan could decrease the drug toxicity and simultaneously increase its solubility rate (9). Most studies applied nanoformulation of AK synthesized by ionic gelation method for leishmaniasis treatment (7$10,12,13,15,16)$. The results of in vitro tests (promastigote and amastigote) showed that AK nanodrug was not very effective in parasite killing. However, they demonstrated an only negligible effect on inhibiting parasite in vivo environment, which is possibly due to a low dose of AK1 mg/kg (7- 


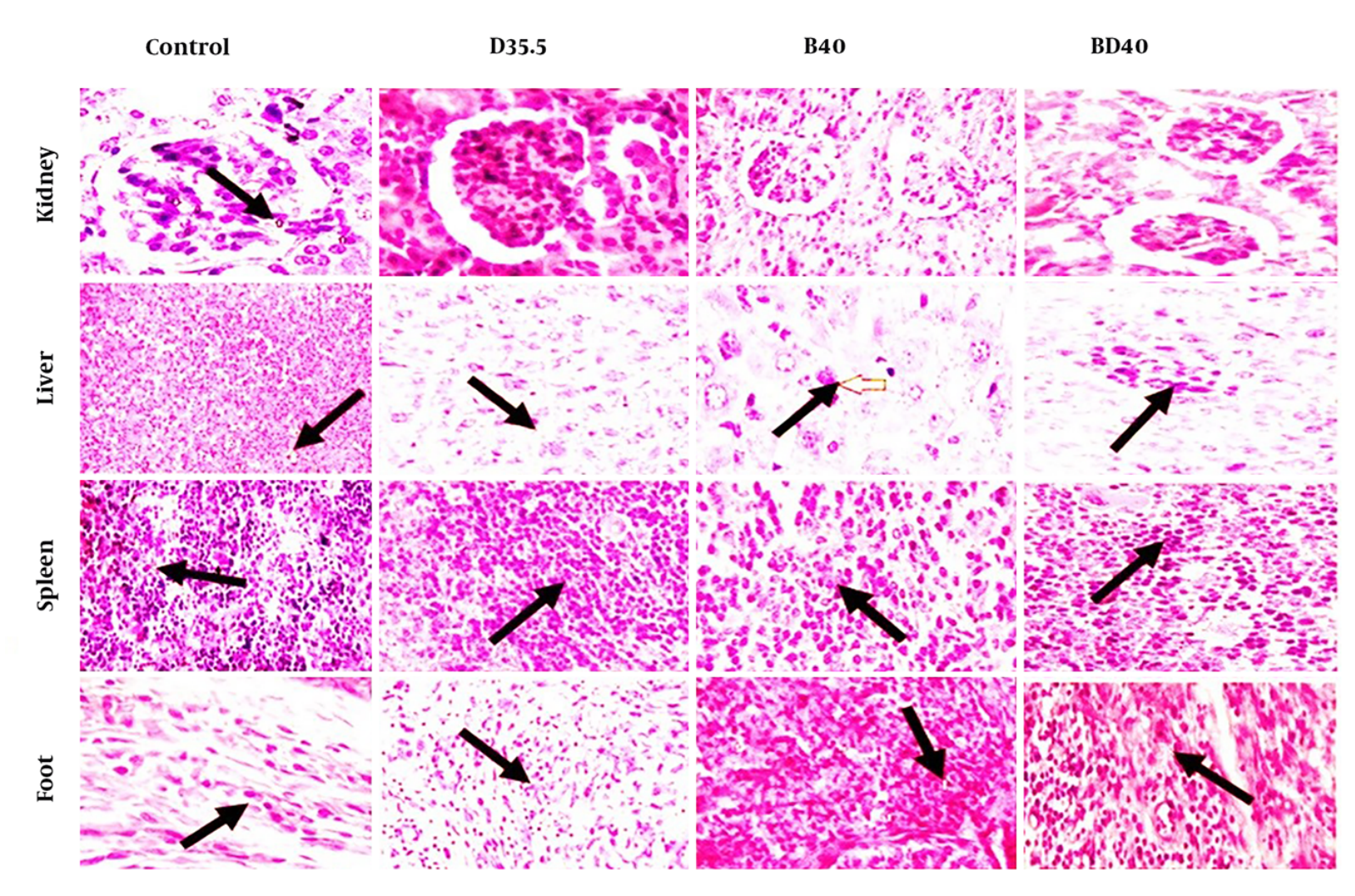

Figure 3. The results of H\&E staining for measurement of parasite number. The black arrows showed amastigotes of $L$. major in kidney, liver, spleen, and footpad of mice treated with $D 35.5, B 40, B D 40 \mathrm{mg} / \mathrm{kg}$, and control group. $(60 \times$ magnification $)$.

$10,12,13,15,16,24)$. In the present study, $A$ was loaded into $K$ synthesized by phase separation method (different from previous studies by using ionic gelation) and it was found that $K$ decreased the toxicity effects of $A$ and increased its solubility $(14,18)$. As in previous studies, AK was not effective in inhibiting parasite in vivo in current dosage (1 $\mathrm{mg} / \mathrm{kg}$ ), then we increased the therapeutic dose of AK to 10 $\mathrm{mg} / \mathrm{kg}$ by using phase separation method and a novel solvent (14). Our findings revealed that parasites were significantly inhibited in our mice model. This success was resulted from increasing the effective therapeutic dose of A to $10 \mathrm{mg} / \mathrm{kg}$ and $\mathrm{K}$ properties including its antileishmanial, immunomodulatory, and wound healing effects $(10,25)$.

On the other hand, as B is a new compound with antileishmanial property, for the first time we considered to load B into a carrier such as $\mathrm{K}$. This formulation increased solubility and decreased the toxicity, additionally, BK formulation was remarkably and significantly more effective on parasite inhibition in dosage of $20 \mathrm{mg} / \mathrm{kg}$ in comparison to our other formulations in recovery of L. major's pathological effects (14).

Dendrimer is the other nanocarrier, which is used fre- quently for its antileishmanial characteristics as positive charge D (PAMAM). For the first time, we used a different kind of D, negative charged ALGD dendrimer was synthesized by using divergent method due to lower price and better availability. We found that ALGD decreased the toxicity effects of A and B, besides increased its solubility slightly more than positive charge $\mathrm{D}(14,18)$. Then, in vivo therapeutic effects of $\mathrm{AD}$ and $\mathrm{BD}$ were evaluated for parasite burden. The results showed that $A D$ and $B D$, in the dosage of 50 $\mathrm{mg} / \mathrm{kg}$ and $40 \mathrm{mg} / \mathrm{kg}$ retrospectively, were more effective in parasite inhibition compared to the previous studies due to using a higher therapeutic dose $(6,11,17)$.

Pathologic findings are important for confirming the in vivo results. For instance Cole et al., confirmed the therapeutic effects of nanodisk containing A by pathological analyses including measurement of parasite number and organs size (26).

In conclusion, we evaluated the therapeutic effects of four nanodrugs and compared them by measurement of organs size (liver, kidney, spleen, and footpad), parasite number, and blood tests on L. major lesions. Our findings indicated that the therapeutic dose of AK $10 \mathrm{mg} / \mathrm{kg}$ and BK 
$20 \mathrm{mg} / \mathrm{kg}$ were more effective than other formulations in recovery of $L$. major's pathological effects without any toxicity. Therefore, overall, it can be concluded that AK and BK, in this mentioned dosage, were considered as a novel candidate for the treatment of leishmaniasis.

\section{Acknowledgments}

This study was resulted from a Ph.D. thesis from Pasteur Institute of Iran. We would like to thank the Pasteur Institute of Iran for providing the grant funding.

\section{References}

1. Shirzadi MR, Mollalo A, Yaghoobi-Ershadi MR. Dynamic relations between incidence of zoonotic cutaneous leishmaniasis and climatic factors in Golestan province, Iran. J Arthropod Borne Dis. 2015;9(2):14860. [PubMed: 26623427]. [PubMed Central: PMC4662787].

2. Saghafipour A, Vatandoost H, Zahraei-Ramazani AR, Yaghoobi-Ershadi MR, Jooshin MK, Rassi Y, et al. Epidemiological study on cutaneous leishmaniasis in an endemic area, of Qom province, central Iran. J Arthropod Borne Dis. 2017;11(3):403-13. [PubMed: 29322057]. [PubMed Central: PMC5758636].

3. Zulfiqar B, Shelper TB, Avery VM. Leishmaniasis drug discovery: Recent progress and challenges in assay development. Drug Discov Today. 2017;22(10):1516-31. doi: 10.1016/j.drudis.2017.06.004. [PubMed: 28647378].

4. Meira CS, Barbosa-Filho JM, Lanfredi-Rangel A, Guimaraes ET, Moreira $\mathrm{DR}$, Soares MB. Antiparasitic evaluation of betulinic acid derivatives reveals effective and selective anti-Trypanosoma cruzi inhibitors. Exp Parasitol. 2016;166:108-15. doi: 10.1016/j.exppara.2016.04.007. [PubMed: 27080160].

5. Bruni N, Stella B, Giraudo L, Della Pepa C, Gastaldi D, Dosio F. Nanostructured delivery systems with improved leishmanicidal activity: A critical review. Int J Nanomedicine. 2017;12:5289-311. doi: 10.2147/IJN.S140363. [PubMed: 28794624]. [PubMed Central: PMC5536235].

6. Jain K, Verma AK, Mishra PR, Jain NK. Surface-engineered dendrimeric nanoconjugates for macrophage-targeted delivery of amphotericin B: Formulation development and in vitro and in vivo evaluation. Antimicrob Agents Chemother. 2015;59(5):2479-87. doi: 10.1128/AAC.0421314. [PubMed: 25645852]. [PubMed Central: PMC4394771].

7. Jain V, Gupta A, Pawar VK, Asthana S, Jaiswal AK, Dube A, et al. Chitosan-assisted immunotherapy for intervention of experimental leishmaniasis via amphotericin B-loaded solid lipid nanoparticles. Appl Biochem Biotechnol. 2014;174(4):1309-30. doi: 10.1007/s12010-0141084-y. [PubMed: 25106894].

8. Singh PK, Pawar VK, Jaiswal AK, Singh Y, Srikanth CH, Chaurasia M, et al. Chitosan coated PluronicF127 micelles for effective delivery of Amphotericin B in experimental visceral leishmaniasis. Int J Biol Macromol. 2017;105(Pt 1):1220-31. doi: 10.1016/j.ijbiomac.2017.07.161. [PubMed: 28780414].

9. Ribeiro TG, Franca JR, Fuscaldi LL, Santos ML, Duarte MC, Lage PS, et al. An optimized nanoparticle delivery system based on chitosan and chondroitin sulfate molecules reduces the toxicity of amphotericin B and is effective in treating tegumentary leishmaniasis. Int J Nanomedicine. 2014;9:5341-53. doi: 10.2147/IJN.S68966. [PubMed: 25429219]. [PubMed Central: PMC4242406].

10. Tripathi P, Jaiswal AK, Dube A, Mishra PR. Hexadecylphosphocholine (Miltefosine) stabilized chitosan modified Ampholipospheres as prototype co-delivery vehicle for enhanced killing of L. donovani. Int JBiol Macromol. 2017;105(Pt 1):625-37. doi: 10.1016/j.ijbiomac.2017.07.076. [PubMed: 28716750].
11. Jain K, Verma AK, Mishra PR, Jain NK. Characterization and evaluation of amphotericin B loaded MDP conjugated poly(propylene imine) dendrimers. Nanomedicine. 2015;11(3):705-13. doi 10.1016/j.nano.2014.11.008. [PubMed: 25596078].

12. Tripathi P, Dwivedi P, Khatik R, Jaiswal AK, Dube A, Shukla P, et al. Development of 4-sulfated $\mathrm{N}$-acetyl galactosamine anchored chitosan nanoparticles: A dual strategy for effective management of Leishmaniasis. Colloids Surf B Biointerfaces. 2015;136:150-9. doi 10.1016/j.colsurfb.2015.08.037. [PubMed: 26381698].

13. Shahnaz G, Edagwa BJ, McMillan J, Akhtar S, Raza A, Qureshi NA, et al. Development of mannose-anchored thiolated amphotericin B nanocarriers for treatment of visceral leishmaniasis. Nanomedicine (Lond). 2017;12(2):99-115. doi: 10.2217/nnm-2016-0325. [PubMed: 27879160]. [PubMed Central: PMC5144491].

14. Mehrizi TZ, Ardestani MS, Khamesipour A, Hoseini MHM, Mosaffa $\mathrm{N}$, Anissian A, et al. Reduction toxicity of Amphotericin B through loading into a novel nanoformulation of anionic linear globular dendrimer for improve treatment of leishmania major. J Mater Sci Mater Med. 2018;29(8):125. doi: 10.1007/s10856-018-6122-9. [PubMed: 30056571]

15. Asthana S, Jaiswal AK, Gupta PK, Pawar VK, Dube A, Chourasia MK. Immunoadjuvant chemotherapy of visceral leishmaniasis in hamsters using amphotericin B-encapsulated nanoemulsion template-based chitosan nanocapsules. Antimicrob Agents Chemother. 2013;57(4):171422. doi: 10.1128/AAC.01984-12. [PubMed: 23357762]. [PubMed Central: PMC3623311].

16. Gupta PK, Jaiswal AK, Asthana S, Verma A, Kumar V, Shukla P, et al. Self assembled ionically sodium alginate cross-linked amphotericin B encapsulated glycol chitosan stearate nanoparticles: Applicability in better chemotherapy and non-toxic delivery in visceral leishmaniasis. Pharm Res. 2015;32(5):1727-40. doi:10.1007/s11095-014-1571-4. [PubMed: 25425053].

17. Daftarian PM, Stone GW, Kovalski L, Kumar M, Vosoughi A, Urbieta $\mathrm{M}$, et al. A targeted and adjuvanted nanocarrier lowers the effective dose of liposomal amphotericin B and enhances adaptive immunity in murine cutaneous leishmaniasis. J Infect Dis. 2013;208(11):191422. doi: 10.1093/infdis/jit378. [PubMed: 23901083]. [PubMed Central: PMC3814840].

18. Yue H, Wei W, Yue Z, Lv P, Wang L, Ma G, et al. Particle size affects the cellular response in macrophages. Eur J Pharm Sci. 2010;41(5):650-7. doi: 10.1016/j.ejps.2010.09.006. [PubMed: 20870022].

19. Aguilar Torrentera F, Lambot MA, Laman JD, Van Meurs M, Kiss R Noel JC, et al. Parasitic load and histopathology of cutaneous lesions, lymph node, spleen, and liver from BALB/C and C57BL/6 mice infected with Leishmania mexicana. Am J Trop Med Hyg. 2002;66(3):2739. [PubMed: 12139220].

20. Chowdhury AR, Mandal S, Goswami A, Ghosh M, Mandal L, Chakraborty D, et al. Dihydrobetulinic acid induces apoptosis in Leishmania donovani by targeting DNA topoisomerase I and II: Implications in antileishmanial therapy. Mol Med. 2003;9(1-2):26-36. [PubMed: 12765337]. [PubMed Central: PMC1430381].

21. Dash SK, Dash SS, Chattopadhyay S, Ghosh T, Tripathy S, Mahapatra SK, et al. Folate decorated delivery of self assembled betulinic acid nano fibers: A biocompatible anti-leukemic therapy. RSC Advances. 2015;5(31):24144-57. doi: 10.1039/c5ra01076d.

22. Liu Y, Gao D, Zhang X, Liu Z, Dai K, Ji B, et al. Antitumor drug effect of betulinic acid mediated by polyethylene glycol modified liposomes. Mater Sci Eng C Mater Biol Appl. 2016;64:124-32. doi: 10.1016/j.msec.2016.03.080. [PubMed: 27127036].

23. Saneja A, Kumar R, Singh A, Dhar Dubey R, Mintoo MI, Singh G, et al. Development and evaluation of long-circulating nanoparticles loaded with betulinic acid for improved anti-tumor efficacy. Int J Pharm. 2017;531(1):153-66. doi: 10.1016/j.ijpharm.2017.08.076. [PubMed: 28823888]. 
24. Ribeiro TG, Chavez-Fumagalli MA, Valadares DG, Franca JR, Rodrigues LB, Duarte MC, et al. Novel targeting using nanoparticles: an approach to the development of an effective antileishmanial drug-delivery system. Int J Nanomedicine. 2014;9:87790. doi: 10.2147/IJN.S55678. [PubMed: 24627630]. [PubMed Central: PMC3931713].

25. Ribeiro TG, Chavez-Fumagalli MA, Valadares DG, Franca JR, Rodrigues $\mathrm{LB}$, Duarte $\mathrm{MC}$, et al. Novel targeting using nanoparticles: An approach to the development of an effective antileishmanial drug-delivery system. Int J Nanomedicine. 2014;9:877-
90. doi: 10.2147/IJN.S55678. [PubMed: 24627630]. [PubMed Central: PMC3931713].

26. Dash M, Chiellini F, Ottenbrite RM, Chiellini E. Chitosan-A versatile semi-synthetic polymer in biomedical applications. Prog Polym Sci. 2011;36(8):981-1014. doi: 10.1016/j.progpolymsci.2011.02.001.

27. Cole P, Bishop J, Beckstead J, Titus R, Ryan R. Effect of Amphotericin B nanodisks on Leishmania major infected mice. Pharm Anal Acta. 2014;5. doi: 10.4172/2153-2435.1000311. [PubMed: 25584195]. [PubMed Central: PMC4288788]. 